\title{
European Marine Observation Data Network - EMODnet Physics
}

\author{
Novellino Antonio ${ }^{1}$, D'Angelo Paolo ${ }^{1}$, Benedetti Giacomo ${ }^{1}$, Manzella Giuseppe ${ }^{1}$, Gorringe Patrick ${ }^{3}$, \\ Schaap Dick ${ }^{2}$, Pouliquen Sylvie ${ }^{4}$, Rickards Lesley ${ }^{5}$
}

${ }^{1}$ ETT SpA, Genoa, Italy.

${ }^{2}$ MARIS, Voorburg, Netherlands.

${ }^{3}$ EuroGOOS AISBL, Brussels, Belgium.

4 IFREMER, Brest, France.

${ }^{5}$ NERC BODC, Liverpool, Merseyside, England.

\begin{abstract}
:
The EMODnet - Physics is a Marine Observation and Data Information System that provides a single point of access to near real time and historical achieved data. It is includes systems for physical data from the whole Europe (wave height and period, temperature of the water column, wind speed and direction, salinity of the water column, horizontal velocity of the water column, light attenuation, and sea level) as monitored by fixed stations, ARGO floats, drifting buoys, gliders, and ferry-boxes. It does provide discovering of data sets (both near real time and historical data sets), viewing and free and open downloading of the data from more than 4400 platforms. Moreover, the system provides full interoperability with third-party software through WMS service, Web Service and Web catalogue in order to exchange data and products according to the most recent interoperability standards. Recent developments ensure the compatibility to the OGS-SWE (Sensor Web Enablement) standard for the description of sensors and related observations using OpenGIS specifications (SensorML, O\&M, SOS).
\end{abstract}

Keywords : EMODnet, Marine Data Management, Data Portal, Open Access, Physical parameters

\section{Introduction}

During the decades, a wealthy amount of marine environmental data have been collected in situ or sensed remotely using different sensors and platforms. All data have a paramount importance at a scientific and environmental levels, as well as at economical level. The high temporal and spatial variability in the sea makes any data irreproducible, as a consequence, the use, preservation and reuse of data pose a lot of challenges, considering that the environmental data are being used by a broader community than even before in Earth Science, environmental studies and assessment.

The new alliance between Earth Science and Information Technology has been beneficial to the society, transforming the data-centric concepts and architectures existing few years ago, into Service- 
Oriented Architectures (SOA) [1]. This change has also involved the concepts of data storage, giving a major importance to data preservation for future use.

In 2010, the European Commission launched the Marine Knowledge program, containing the important message that innovation in blue economy is 'held back by a lack of information about the sea' [2]. It is important to underline that the re-use of data can generate new science and also new services for the civil society. The preservation of data, that is impossible to recreate and that have been expensively generated, is a necessary step, inter alia, to assess compliance with legal requirements.

The European Marine Observation and Data Network (EMODnet) has been established by the European Commission to bring together marine data, data products and metadata from diverse sources within Europe in a uniform way. The EMODnet web portals provide access to specific thematic data sets. 
The ongoing EMODnet Physics has the objective to provide access to archived and near real time data on physical conditions in European seas and oceans from fixed monitoring stations and ferrybox lines and to determine how well the data meets the needs of users.

The specific objectives of the EMODnet Physics are:

- Make available and accessible existing data from public and private organizations relating to the state of sea basins; processing them into interoperable formats which includes agreed standards, common baselines or reference conditions; assessments of their accuracy and precision;

- improve, operate and maintain the EMODnet Physics portal allowing public access and viewing of data, metadata and data products (in accordance with INSPIRE recommendations [3]).

The present paper represents a development of the MTSIEEE OCEANS2015 minute.

\section{EMODNET PHYSICS}

The EMODnet Physics portal gives access to two major data streams:

1. Near-real-time (within 24 hours from acquisition) data collected for operational needs (e.g. moored buoys, rigs/platforms, coastal stations) and by automatic observatories at sea (e.g. profiling floats, drifting buoys, ferrybox, ships of opportunity, research vessels) which are transmitted in near real-time to the shore, and

2. Archived data derived from further elaboration and validation of the NRT data.

The access to the first data stream is ensured by the Regional Operational Systems of EuroGOOS (ROOSs) and in particular by the In Situ Thematic Assembling Center (MyO IS TAC) system; the second data stream is relying on the National Oceanographic Data Centres (NODCs) network organised by the SeaDataNet European project. The NODCs are in charge for ocean data validation, quality check and update for marine environmental monitoring.

The portal is providing different sections: 1) the landing page www.emodnet-physics.eu/portal that offers a detailed description of the activities, the contributors, the standards, the background, 2) the map page www.emodnet-physics.eu/map that is the operational core engine of the system, in fact here the user can access all available data, customize the map visualization and set different display layers. It is also possible to interact with all the information on the map using the filters provided by the service that can be used to select the stations of interest depending on the type, physical parameters measured, the time period of the observations in the database of the system, country of origin, the water basin of reference. It is also possible to browse the data in time using the slider in the lower part of the page that allows the user to view the stations that recorded data in a particular time period.

Finally, it is possible to change the standard map view with different layer that provide additional visual information on the status of the waters.

The platform panel available from the main map by clicking on a single platform, provides information on the measurements carried out by the station. Moreover, the system provides full interoperability with third-party software through WMS service, Web Service and Web catalogue in order to exchange data and products according to the most recent interoperable standards www.emodnet-physics.eu/map/dashboard .

Further developments will ensure the compatibility to the OGS-SWE (Sensor Web Enablement) standard for the description of sensors and related observations using OpenGIS specifications (SensorML, O\&M, SOS).

The result is an excellent example of innovative technologies for providing open and free access to georeferenced data for the creation of new advanced (operational) oceanography services.

\section{EMODNET DATA CATEGORIES}

EMODnet Physics makes available near real time data and metadata provided by national data originators organized at EuroGOOS Regional level according the ROOSs infrastructure. It provides free and open access to all available near real time data of the latest 60 days to any user without the need for registration. 1 and Fig. 1 shows the operational platforms that are part of the EMODnet Physics system. Operational platforms provide data time series as soon as data is ready, for example a fixed platform is delivering data daily, an ARGO float is delivering almost weekly. 
To access data older than 60 days (monthly archives) however, users need to register and $\log$ in to access the system.

Validated delayed data series and metadata are organized according to, and in collaboration with, the network of NODCs. By the end of 2014, EMODnet Physics fully integrated historical data datasets for 794 platforms (11450 datasets) which are made available via the SeaDataNet Common Data Index (CDI) discovery and request system [4].

TABLE I. Number OF PLATFORMS CONNECTED TO EMODNET PHYSICS

\begin{tabular}{|l|c|}
\hline \multicolumn{1}{|c|}{ Platform names/system } & Number of platforms \\
\hline Drifting Buoys & 2393 \\
\hline Ferrybox & 17 \\
\hline Gliders & 13 \\
\hline $\begin{array}{l}\text { Fixed moorings or mooring time } \\
\text { series }\end{array}$ & $781(+794)$ \\
\hline Profiling Floats, Vertical Profiles & 112 \\
\hline Argo Floats & 1096 \\
\hline TOTAL & 4412 \\
\hline
\end{tabular}

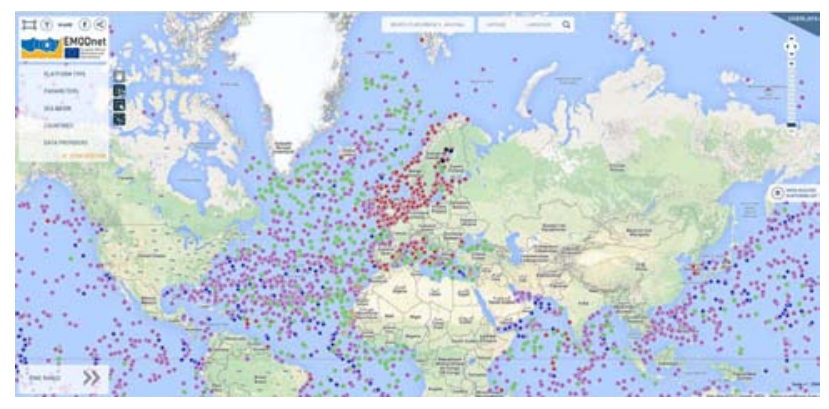

Fig. 1: EMODnet Physics spatial data distribution. Fixed stations are shown in red, drifting buoys in violet, gliders in yellow, profilers in blue, ARGO floats in green and ferryboxes in black.

\section{INTEROPERABILITY CONCEPTS}

Four layers of different abstraction levels of interoperability can be defined at the base of EMODnet Physics infrastructure model:

- Technical/basic: this layer is implemented at each data source through internet connection and basic services for data transfer and browsing (e.g FTP, HTTP, etc).

- Syntactic: allowing the interchange of metadata and protocol elements. This layer corresponds to a definition Core Metadata Set, the format of exchange/delivery for the data and associated metadata and possible software. This layer is implemented by adopting INSPIRE compliant metadata set and common data formats.
- Functional/pragmatic: based on a common set of functional primitives or on a common set of service definitions. This layer refers to the definition of services based on Web services standards. This layer is implemented by the adoption of INSPIRE compliant network services.

- Semantic: allowing to access similar classes of objects and services across multiple sites. This layer corresponds to portal interface, terminology and thesaurus.

The EMODnet Physics system is composed by two basic sub-layers that can be called "Data Management" layer and "Network" layer as depicted in Fig. 2.

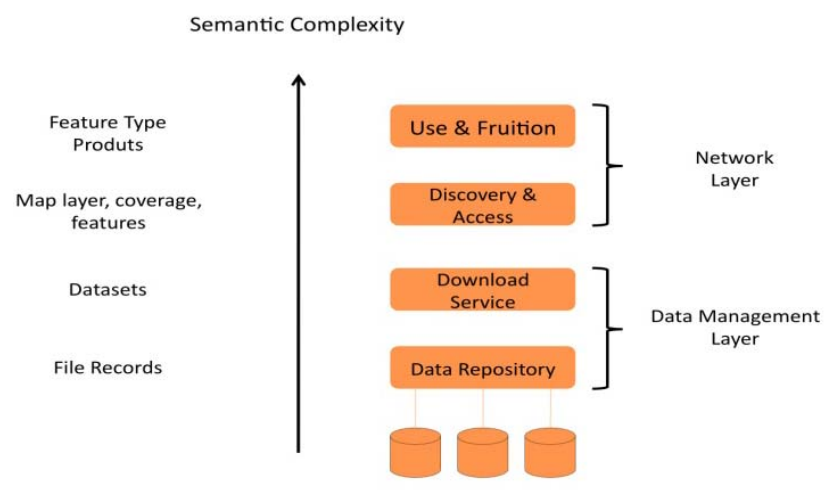

Fig. 2: EMODnet Physics services layers and associated semantics

\section{Data management layer}

This layer provides:

- MetaInformation (data management issues, protocols, QA/QC, instruments, etc; INSPIRE and ISO compliance)

- Format/Convention (INSPIRE and ISO models)

- Digital Products Repositories (archival technology)

The metadata must contain all associated information needed to complete the data itself (date, source, ... ). This layer includes all issues on data quality. Information on protocols and practices are made available to users.

The data exchange is designed by defining protocols that normalise the data exchanges.

\section{Network layer}

This layer includes:

- Access Services (WMS, OPeNDAP, WFS, ...)

- Catalogues (THREDDS, CDI, RDF, CSW, ...)

- Mediation/Federation

- User interfaces 
The functional requirements of these components are:

Discoverability: How users find products, tools to read or use the data.

Access management: Ownership and rights management are taken into account.

Selection: Criteria applied when selecting products.

Complexity: The products are composite in nature, with links to external objects and external dependencies (such as calibration information, quality assessment and quality control procedures), and are highly complex in structure.

\section{INTEROPERABILITY SERVICES}

EMODnet Physics provides a range of interoperability services for machine-to-machine communication for easier data integration and accessibility. Core services include the provision of web services, Web Map Service (WMS) and Web Feature Service (WFS). EMODnet Physics is exposing fully OGC compliant WMS/WFS layers by exploiting a GeoServer based infrastructure. By linking to these interoperability services many users are already adding EMODnet Physics data into their own applications and products. For example, these services allow an easy uptake of EMODnet Physics datasets into the Ocean Data Portal (ODP) from the International Oceanographic Data and Information Exchange (IODE) programme of UNESCO's Intergovernmental Oceanographic Commission (IOC) giving the global visibility to the EMODnet physics data providers.

\section{ACCESS PROVISION MODALITIES AND SERVICES}

The EMODnet Physics Portal consists of two main components: (i) a landing page with general information on the project approach, available data and products from connected platforms as well as the most important and widely adopted quality control procedures; and (ii) a set of services providing user interfaces and functionalities to search, visualise and retrieve data and products.

The main services include (i) a map viewer to search, visualise and retrieve data and products; (ii) a catalogue which provides similar functionalities to the map viewer but in a more text based interface without visualising the platform locations on a map; (iii) a range of interoperability services for machine-to-machine communication.

\section{The EMODnet Physics map viewer}

The EMODnet Physics map viewer is designed to facilitate data discovery and selection. The map viewer visualises the location of platform connected to the system. For moving platforms the latest position is shown. The viewer provides filters to allow users to identify and select a subset of available data based on the type of platform (e.g. fixed stations, drifting buoys, etc.), physical parameter (e.g. sea temperature, sea level, waves and winds), sea basin, country and data provider. By selecting a platform, a small panel summarizes what the platform is providing (parameters and latest measurements) as well as some basic platform information such as platform owner.

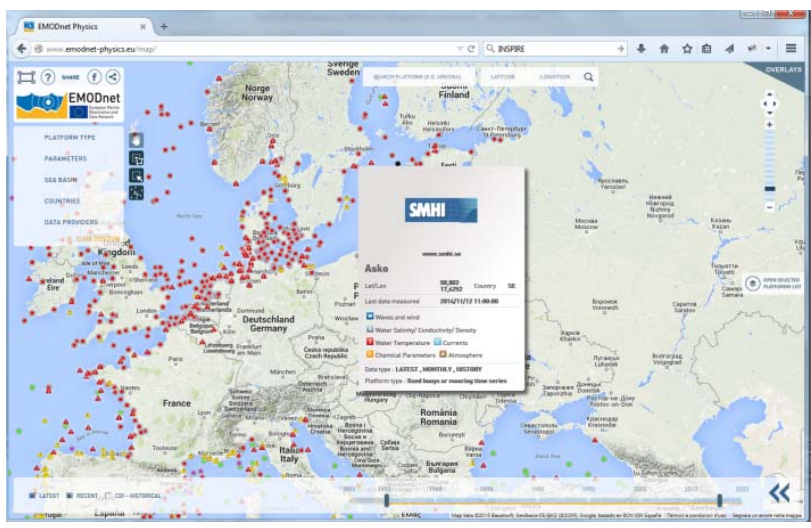

Fig. 3: EMODnet Physics map viewer showing a selected platform summary panel.

The map viewer also provides an option to select the "time range" allowing users to define a window in time to obtain an overview of the platforms providing data in the selected time period. There are basically three categories of data sets depending on the type of data providers: (i) 'latest data' refers to platforms which provide at least one dataset for the past 60 days; (ii) 'recent data' relates to all platforms which are still operational but not necessarily providing datasets in the last 60 days; while (iii) 'CDI-historical' refers to platforms with datasets validated by national oceanographic data centres.

But the map viewer does more than that: by clicking on a specific platform a dedicated platform page is provided which allows user to easily see available parameters, plot 
previews, see data availability, provided products (monthly averages, max and min products, and if available wind products) and available validated datasets. The platform page also provides a daily digest service which allows users to register for email updates on a daily base with the latest values of measured parameters for selected platforms.

\section{The EMODnet Physics catalogue}

The catalogue provides in a more organised way the list of platforms with their WMO codes. These are unique and persistent identifiers for an environmental monitoring facility (station/platform).

\section{Machine-to-machine communication}

This is an important element of EMODnet Physics, since allow to be interoperable with other international initiatives, such as the Ocean Data Portal of the IODEIOC-UNESCO (Fig. 4).

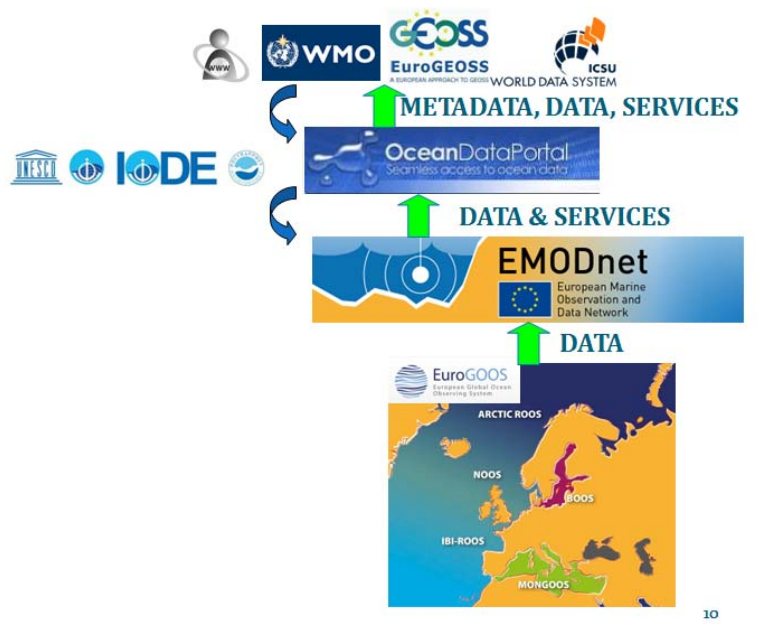

Fig.4: Machine-to-machine communication

\section{CONCLUSIONS}

EMODnet is a long-term marine data initiative supporting a sustainable blue economy in Europe, constructed through a stepwise approach. Halfway through its development, the resources and services are already useful and data portals are progressing rapidly to (i) become fully operational; (ii) provide the best available data, free of restrictions on use; and (iii) become more user friendly and fit for purpose. EMODnet Physics is a perfect example of how EMODnet, through the application of innovative technologies, can act as a gateway and one stop shop to access the vast resources of geo-referenced marine data in Europe with added value tools and functionality.

Bringing observations, products, services and knowledge to users and the public requires appropriate tools and guidance. Ensuring fitness for purpose can only be done together with a growing number of data providers and users and EMODnet will increasingly rely on the involvement of stakeholders to guide further developments.

Since its inception in 2011, the EMODnet Physics portal has attracted a steadily increasing number of visitors and users, greatly assisted by a dedicated communication strategy and a user-oriented approach. For example, the portal infrastructure automatically sends e-mails to more than 1000 users whenever a news item is published and users are updated about new datasets matching their interests when they are made available. As a result, by the end of 2014 each month the portal receives about 600 unique visitors and more than 20000 physics data downloads are requested monthly (partly manual partly through machine to machine communication). By providing access to physical data and metadata, EMODnet Physics also contributes to important global initiatives such as the Ocean Data Portal (ODP) and the Global Monitoring for Environment and Security (GMES) marine core service.

EMODnet Physics is under continuous development to improve the streamlining and optimization of the data flow, to gradually extend the provided features and strengthen the backoffice infrastructure to ensure data and information remains only one click away from the user. Future developments will include closer collaboration with the marine component of the EU's Copernicus earth observation programme and wider coverage of underrepresented sea-basins with a specific target to fill gaps in time series.

\section{ACKNOWLEDGEMENTS}

The European Marine Observation and Data Network (EMODnet) is financed by the European Union under Regulation (EU) No. 1255/2011 of the European Parliament and of the Council of 30 November 2011 establishing a Programme to support the further development of an Integrated Maritime Policy. 


\section{REFERENCES}

[1] M. H. Valipour, B. A. Zafari, Kh. N. Maleki and N. Daneshpour, 'A Brief Survey of Software Architecture Concepts and Service Oriented Architecture', in Proceedings of 2nd IEEE International Conference on Computer Science and Information Technology, ICCSIT'09, pp 34-38, Aug 2009, China.

[2] Communication from the Commission: Innovation in the Blue Economy: realising the potential of our seas and oceans for jobs and growth - $\operatorname{COM(2014)~254/2~}$ $(13 / 05 / 2014)$

[3] Directive 2007/2/EC of the European Parliament and of the Council of 14 March 2007 establishing an Infrastructure for Spatial Information in the European Community (INSPIRE)

[4] S. Nativi, P. Mazzetti, M. Santoro, E. Boldrini, G. M. R. Manzella and D. M. A. Schaap, 'CDI/THREDDS interoperability in the SeaDataNet framework' Adv. Geosci., 28, 17-27, 2010 doi:10.5194/adgeo-28-172010 